\title{
Color-relation-based capture occurs globally
}

\author{
Huimin Hua ${ }^{1,2} \cdot$ Jie Zhang ${ }^{1,2} \cdot$ Yanju $\mathrm{Li}^{1,2} \cdot$ Feng $\mathrm{Du}^{1,2}$
}

Published online: 5 March 2019

(C) The Psychonomic Society, Inc. 2019

\begin{abstract}
Previous studies have shown that a distractor matching the target-nontarget color relation did not capture attention when it appeared outside of the attentional window, indicating that color-relation-based attention is lack of global selection. The present study examined whether a distractor best matching the target-nontarget color relation instead of the target color captured attention outside of the attentional window. The results consistently showed that peripheral distractors best matching the target-nontarget color relation instead of the target color captured attention outside of the attentional window. Additionally, color-relation-matched distractors outweighed target-color-matched distractors in capturing attention under this circumstance. This suggests that the color-relation-based attention shares the hallmark of global selection with the color-based attention. Combined with the previous finding of double dissociation of color-relation-based attention and color-based attention (Du \& Jiao, Journal of Experimental Psychology: Human Perception and Performance, 42(4), 480-493, 2016; Du, Yin, Qi, \& Zhang, Psychonomic Bulletin \& Review, 21(4), 1011-1018, 2014), preference for color-relation within the attentional window and priority for target color outside of the attentional window might be a strategic choice of visual attention.
\end{abstract}

Keywords Attentional capture $\cdot$ Relation-based attention $\cdot$ Attentional window

Attention is crucial for selecting relevant information from crowded visual stimuli. Researchers have proposed that visual attention is either captured in a purely stimulus-driven fashion or guided by top-down attentional control setting. For example, a color singleton captures attention in a purely stimulusdriven manner (Theeuwes, 1991). In contrast, it has been convincingly shown that people can selectively process stimuli based on features that match our current goal (Du \& Abrams, 2008; Eimer \& Kiss, 2008; Folk \& Remington, 1998), which has been labeled as feature-based attention. For example, Folk and colleagues found that irrelevant cues captured attention only if they matched the target-defining feature - onset or color in that case (Folk, Remington, \& Johnston, 1992). Folk and colleagues initially proposed that attention is contingent upon target domains such as color or onset (e.g., Folk, Remington,

Feng $\mathrm{Du}$

duf@psych.ac.cn

1 Key Laboratory of Behavioral Science, Institute of Psychology, Chinese Academy of Sciences, 16 Lincui Road, Beijing 100101, China

2 Department of Psychology, University of Chinese Academy of Sciences, Beijing, China
\& Johnston, 1992, 1993). But later they showed that attention can also be set for specific feature values (e.g., Folk \& Remington, 1998). More converging evidence has shown that involuntary capture can rely on a variety of target features, such as a specific color, luminance or orientation of target (Du \& Abrams, 2008, 2012), or a combination of multiple features (Kiss, Grubert, \& Eimer, 2013).

Global selection is the hallmark of feature-based capture or feature-based attention (Du \& Abrams, 2010; Treue \& Martínez Trujillo, 1999). Specifically, when a uniquely colored target letter was embedded in a rapid serial visual presentation (RSVP) stream of nontarget letters at fixation, only peripheral distractors matching target color can involuntarily capture attention, disrupting central target identification (Du \& Abrams, 2008; Folk, Leber, \& Egeth, 2002; Serences et al., 2005). Given the peripheral distractors always appearing outside of the area of interest, the impairment of central target identification, which is contingent upon the target-distractor color match, is referred to as a "spatial blink."

However, recent evidence has shown that the attentional control settings can be selectively tuned to not only a specific target feature value but also a particular target-nontarget size or color relation (Becker, 2010; Becker, Folk, \& Remington, 2010, 2013; Brand, Oriet, Johnson, \& Wolfe, 2014; Harris, 
Remington, \& Becker, 2013; Kiss \& Eimer, 2011; Kiss et al., 2013; Meeter \& Olivers, 2014). For example, when participants were required to search for an orange target among gold nontargets, an irrelevant cue matching the target-nontarget color relation (redder) involuntarily captures attention (e.g., a red cue among orange cues or a gold cue among yellow cues), resulting in a cuing effect. Surprisingly, irrelevant cues that match the target color but not the target-nontarget color relation (e.g., an orange cue among red cues) fail to capture attention (Becker et al., 2013). Additionally, cues that are less similar to the target can cause a greater cuing effect than do cues identical to the target color, as long as the less similar cues are relationally better than the identical cues. Thus, Becker et al. (2013) proposed that the target-nontarget relation is more important than a specific target feature in guiding attention and eye movements, although attention can be tuned to a specific color through training (Becker, Harris, Venini, \& Retell, 2014).

However, these aforementioned studies found no evidence that relation-based attentional capture operates globally. A recent study found that a target-color-matched distractor captured attention outside of the attentional window, but there was no occurrence of size-relation-based capture outside of the attentional window (Du, Yin, Qi, \& Zhang, 2014). They showed that an irrelevant peripheral size distractor that matched the targetnontarget size relation (one distractor was smaller than the others in the periphery) did not capture attention, though the same smaller size cue among others produced a cuing effect. Additionally, the peripheral color distractor matching the target color captured attention outside of the attentional window regardless of the size relation between target and nontarget. Furthermore, Du and Jiao (2016) showed that, when distractors appeared outside of the attentional window, distractors matching the target color rather than the target-nontarget color relation consistently capture attention. In contrast, when distractors appeared within the attentional window, color distractors matching the target-nontarget color relation are more likely to capture attention than are target-color-matched distractors. The double dissociation between color-based capture and color-relationbased capture indicates that the two types of attentional selection have distinctive mechanisms. This finding calls into question whether a target-nontarget relation truly operates globally across the whole visual field as a target feature does. If relation-based capture cannot occur outside of the attentional window, it is fundamentally distinct from feature-based capture, which has been shown to occur outside of the attentional window.

A recent study (Goldstein \& Beck, 2016) showed that a relation-matched distractor can capture attention even in inattention blindness. Researchers asked participants to count the number of times gray letters ( $T$ or $\mathrm{L}$ ) bounced off the sides of a central box while ignoring either white or black letters. In some trials, unexpected objects (UEOs) moved on the display. The UEO could best match the target-distractor relation, but not the target color, or match both the target-distractor relation and the target color, or match the distractor color. Results showed that the UEOs best matching the target-distractor relation were reported the most. For example, when participants monitored the gray letter while ignoring the black letter, a lighter relationship was formed between the target and distractor. Under this circumstance, a white UEO that best matched the target-distractor relation but mismatched target color was reported the most. This finding reflects that UEO in the best relation-matched condition is more likely to capture attention when available attentional resources are scarce. However, unlike the spatial blink task (Du \& Jiao, 2016; Du et al., 2014), there is no stringent spatial control in the inattentional blindness task of Goldstein and Beck's (2016) study. Goldstein and Beck's finding did not address whether relation-based capture can occur outside of the attentional window, and thus cannot support the global selection of relation-based attention.

Therefore, whether relation-based capture can occur outside of the attentional window under some special circumstance is still unknown. If relation-based capture truly cannot occur outside of the attentional window, relation-based attention operates locally rather than globally, indicating it differs fundamentally from feature-based attention. However, if relation-based attentional capture can occur outside of the attentional window under some special circumstances, then the dissociation between relation-based and feature-based capture is more likely to be a strategic choice of visual attention under different circumstances.

\section{Experiment 1}

The present study aims to examine whether, under special circumstances, relation-based capture can appear outside of the attentional window. In order to force participants to adopt a relation-based attentional control setting, we set up three target colors and explicitly instructed participants to search for a redder or greener target letter among nontarget letters in the RSVP. If relation-based capture truly differs from the feature-based capture in the global selection mechanism, then the color-relationbased capture would not occur outside of the attentional window. Otherwise, the relation-based capture should still occur outside of the attentional window when participants were forced to use a relation-based attentional control setting.

\section{Method}

\section{Participants}

Twenty-four students (11 males) participated in this experiment for cash compensation. We estimated the sample size by using the main effect of the distractor color in the redder target block of our pilot study with 20 participants, which was the smallest $\left(\eta_{\mathrm{p}}{ }^{2}=0.255\right)$. The results showed that 19 
participants were needed to achieve a power of 0.95 with $\alpha=$ .05 (using the "as in SPSS" option in G*Power 3.1). Thus, we recruited 24 participants in order to achieve a robust result pattern. All participants had normal or corrected-to-normal vision. The present study was approved by the Institutional Review Board of the Institute of Psychology at the Chinese Academy of Sciences.

\section{Apparatus, stimuli, and procedure}

The stimuli were presented on a 17-inch CRT monitor with a refresh rate of $85 \mathrm{~Hz}$ and a resolution of $1,024 \times 768$ pixels. A chin rest was used to keep the viewing distance fixed at $57 \mathrm{~cm}$. The trial procedure was illustrated in Fig. 1. Each trial began with a presentation of a gray cross in the center of the display for $800 \mathrm{~ms}$, followed by a sequence of 16 uppercase letters. The letters were randomly selected and each letter was presented at the center for $59 \mathrm{~ms}$, followed by a 47-ms blank interval (stimulus-onset asynchrony [SOA] $=106 \mathrm{~ms}$ ). During each trial, the target letter displayed randomly in the eighth through 12th frame of the letter sequence. Participants were asked to focus attention on the centrally presented RSVP stream and search for either a redder or greener target letter in the central stream. The peripheral irrelevant distractor always appeared two frames before that containing the target. Namely, a pound $\operatorname{sign}\left(\#, 0.8^{\circ} \mathrm{W} \times 1.2^{\circ} \mathrm{h}\right.$ ) randomly appeared one of the four distractors spatial positions $\left(5^{\circ}\right.$ above, below, left, and right of the center in the blank interval frames) in the sixth through 10th frame. These peripheral distractors were considered to be outside of the attentional window since they appeared at locations irrelevant to central target.

The experiment included two blocks with different targetnontarget colors relations. In the redder block, the participants were required to report a redder target letter among the greenish-yellow or yellowish-green nontarget letters in the

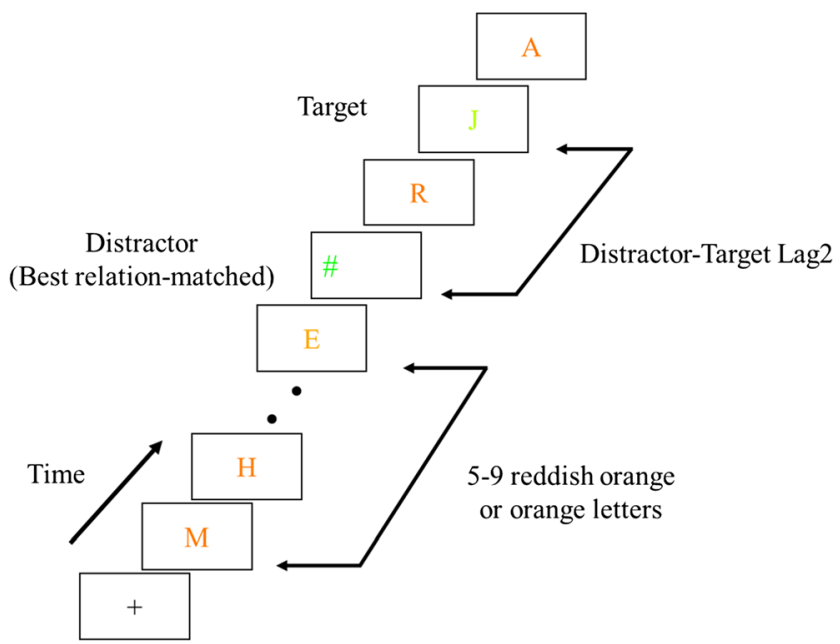

Fig. 1 Schematic of the procedures used in Experiment 1. Participants were asked to report the greener target letter. (Color figure online) central RSVP stream. Figure 2 illustrated the colors of targets, nontargets, and distractors used in this experiment. In the redder block, the RGB values for the three target colors were gold $(255,215,0)$, orange $(255,185,0)$, and dark orange $(255,155$, $0)$. The color of nontarget was either greenish yellow (205, $252,32)$ or yellowish green $(128,251,79)$. The RGB values of distractors were gray $(127,127,127)$, red $(255,75,0)$, green $(0,255,0)$, and target colors.

In the greener block, the target was a greener letter among reddish-orange or orange nontargets in the central RSVP stream. For the greener block, the RGB values of target colors were yellow $(240,255,0)$, green yellow $(210,255,0)$, and bright green $(180,255,0)$. The RGB values of nontarget color were reddish orange $(255,120,0)$ and orange $(255,165,0)$. The distractors were the same as those used in the redder block. Three target colors were randomly chosen for each trial. The order of the two blocks is counterbalanced between participants.

\section{Design}

Each trial was in one of four distractor-color conditions: (1) The neutral condition (the pound sign was gray in color); (2) the best relation-matched condition (a red color pound sign in the redder block or a green color pound sign in the greener block); (3) the target-color-matched condition (the targetcolor-matched distractor was identical to the target color for each trial); (4) the relation-reversed condition (the green color pound sign in the redder block or the red color pound sign in the greener block). The experiment contained 72 replications of each combination of the four distractor-color conditions and two blocks, for a total of 576 trials. The participants took 32 practice trials before each block and had a break after 72 trials in each block.

\section{Results}

A 2 (target-nontarget relation) $\times 4$ (distractor color conditions) ANOVA showed that the main effect of target-

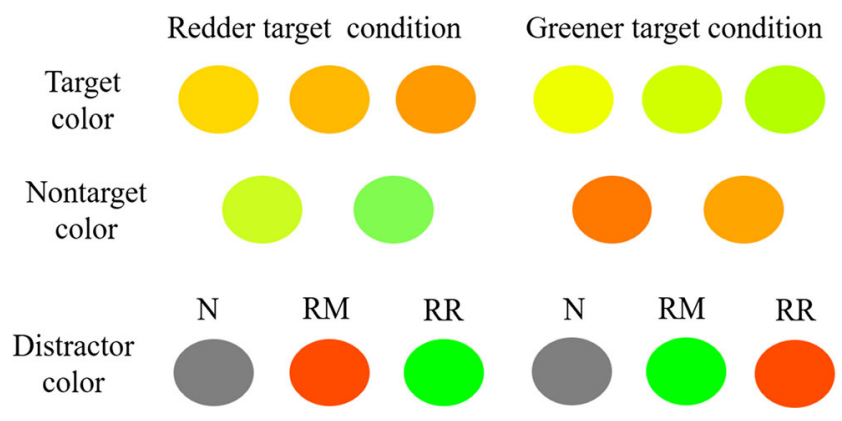

Fig. 2 Colors used in redder and greener block in Experiment 1. $\mathrm{N}=$ neutral condition; $\mathrm{RM}=$ best relation-matched condition; $\mathrm{CM}=$ targetcolor-matched condition; $\mathrm{RR}=$ relation-reversed condition. . Color figure online) 
nontarget relation was significant, $F(1,23)=25.74, p<.001$, $\eta_{\mathrm{p}}{ }^{2}=0.528$, showing that the redder target block had worse performance than the greener target block. The main effect of distractor color conditions was also significant, $F(3,69)=$ $23.75, p<.001, \eta_{\mathrm{p}}{ }^{2}=0.508$. The further simple effect analysis of the distractor color conditions had shown that the best relation-matched condition had lower accuracy than the other three distractor conditions. Specifically, the best relationmatched condition was significantly less accurate than the target-color-matched condition, $t(23)=-5.25, p<.001, d=$ 1.061, the neutral condition, $t(23)=-5.66, p<.001, d=$ 1.155 , and the relation-reversed condition, $t(23)=-6.15, p<$ $.001, d=1.257$. Additionally, the target-color-matched condition was also less accurate than the neutral condition, $t(23)=$ $-3.67, p=.001, d=0.749$, and the relation-reversed condition, $t(23)=-3.36, p=.003, d=0.686$.

The interaction between the two factors was also significant, $F(3,69)=2.89, p=.042, \eta_{\mathrm{p}}{ }^{2}=0.112$. Thus, we further analyzed the effect of distractor color conditions on accuracy separately for the redder and greener target (see Fig. 3a and b, respectively). For the redder target condition, the main effect of distractor color conditions was significant, $F(3,69)=$ $15.45, p<.001, \eta_{\mathrm{p}}{ }^{2}=0.402$. Further pairwise comparison showed that the red distractors best matching the targetnontarget relation caused the lowest accuracy. More specifically, the red distractors were significantly less accurate than the target color distractors (the target-color-matched condition; $p=.003)$, the neutral distractors $(p=.001)$, and the green distractors (the relation-reversed condition; $p<.001$ ). There was no difference between other distractor color conditions (all $p \mathrm{~s}>.05$ ).

For the greener target, the main effect of distractor color conditions was significant, $F(3,69)=14.85, p<.001, \eta_{\mathrm{p}}{ }^{2}=$ 0.392. Further pairwise comparison showed that the accuracy of the green distractor that best matched the target-nontarget relation caused the lowest accuracy. Specially, the green distractor was significantly less accurate than the targetcolor-matched distractors $(p=.002)$, the neutral distractor condition $(p<.001)$, and the red distractor (the relationreversed condition; $p=.010$ ). In addition, the target-color-
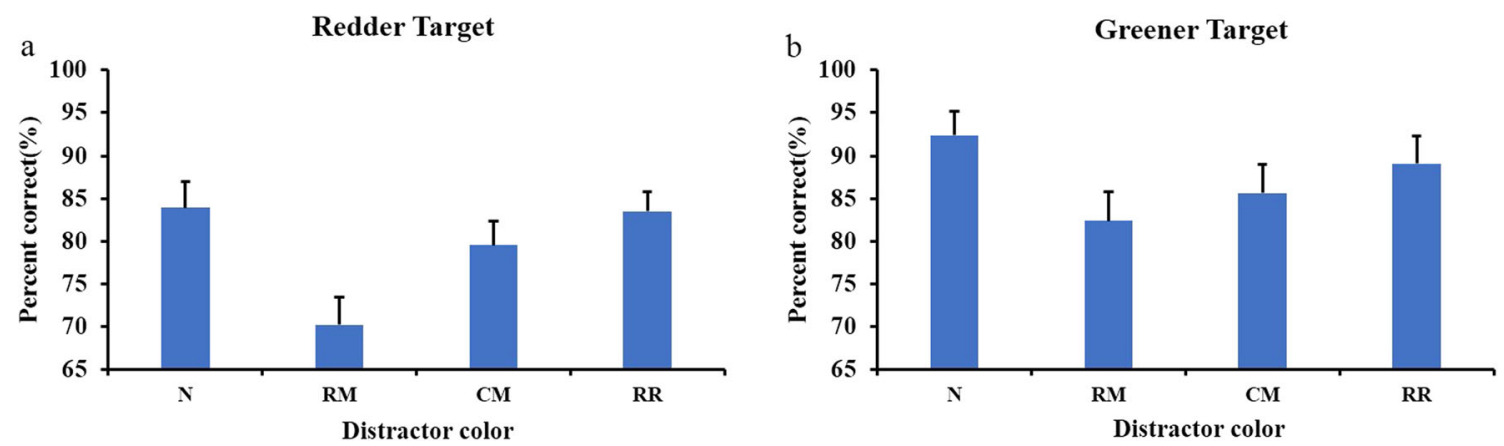

Fig. 3 Accuracy of different distractor conditions for the redder target (a) and greener target (b) in Experiment 1. Error bars represent the standard error. $\mathrm{N}=$ neutral condition; $\mathrm{RM}=$ best relation-matched condition; $\mathrm{CM}=$ target-color-matched condition; $\mathrm{RR}=$ relation-reversed condition 
they were instructed that the red color in redder block and the green color in greener block would never be a target.

Second, the redder block had worse accuracy than the greener block in Experiment 1. It is possible that the contrast between the greenish targets and their distractors is more pronounced than the contract between the redder targets and their distractors. Therefore, we narrowed down the contrast between the target and distractors in the greener block of Experiment 2. The RGB values for present targets in the greener block were yellow $(245,240,0)$, green yellow $(215$, $240,0)$, and bright green $(185,240,0)$. The RGB values for nontarget colors and distractors were same as those in the greener block of Experiment 1.

\section{Results}

A 2 (target-nontarget relation) $\times 4$ (distractor color conditions) ANOVA analysis had shown that the main effect of target-nontarget relation was significant, $F(1,23)=5.14, p$ $=.033, \eta_{\mathrm{p}}^{2}=0.183$, showing that the redder target block had worse performance than the greener target block. Most importantly, the main effect of distractor color conditions was significant, $F(3,69)=16.13, p<.001, \eta_{\mathrm{p}}^{2}=0.412$. The further simple effect analysis of the distractor color conditions had shown that the best relation-matched distractor had lower accuracy than the other three distractors. Specifically, the best relation-matched condition was significantly less accurate than the target-color-matched condition, $t(23)=-5.18, p<$ $.001, d=1.058$, the neutral condition, $t(23)=-4.58, p<$ $.001, d=0.935$, and the relation-reversed condition, $t(23)=$ $-4.35, p<.001, d=0.887$. In addition, the target-colormatched condition was also less accurate than the neutral condition, $t(23)=-2.92, p=.008, d=0.595$, and the relationreversed condition, $t(23)=-2.65, p=.014, d=0.539$. The interaction between the target-nontarget relation and distractor color was not significant, $F(3,69)=2.30, p=$ $.085, \eta_{\mathrm{p}}^{2}=0.091$.

We further analyzed the effect of distractor color on accuracy separately for the redder target and greener target (see Fig. 4a and b, respectively). For the redder target condition, the main effect of distractor color conditions was significant, $F(3,69)=15.93, p<.001, \eta_{\mathrm{p}}{ }^{2}=0.409$. The further pairwise comparison showed that the red distractors that best matched the target-nontarget color relation caused the lowest accuracy. More specifically, the red distractors were significantly less accurate than the target color distractors (the target-color-matched condition; $p<.001)$, the neutral distractors $(p=.002)$, and the green distractors (the relation-reversed condition; $p<$ .001). Additionally, the target-color-matched condition was marginally less accurate than the relation-reversed condition $(p=.074)$. There was no difference between other distractor color conditions (all $p \mathrm{~s}>.05$ ).

For the greener target, the main effect of distractor color conditions was significant, $F(3,69)=9.51, p<$ $.001, \eta_{\mathrm{p}}{ }^{2}=0.293$. The further pairwise comparison showed that the accuracy of the green distractor that best matched the target-nontarget color relation caused the lowest accuracy. Specially, the green distractor was significantly less accurate than the target-color-matched distractors $(p=.014)$, the neutral distractor condition $(p$ $=.005$ ), and the red distractor (the relation-reversed condition; $p=.039$ ). In addition, the target-color-matched distractor condition made worse performance than the neutral distractor condition $(p=.049)$.

\section{General discussion}

Both experiments showed that distractors best matching the target-nontarget color relation caused the lowest accuracy. In addition, the best relation-matched distractors captured attention more than the target-color-matched distractors. This is the first study to show the relation-based attentional capture outside of the attentional window. It indicates a global selection mechanism for relation-based attention. These findings further demonstrate that the dissociation between relation-based attention and feature-based attention might be due to the different default choices of visual attention under different circumstances.
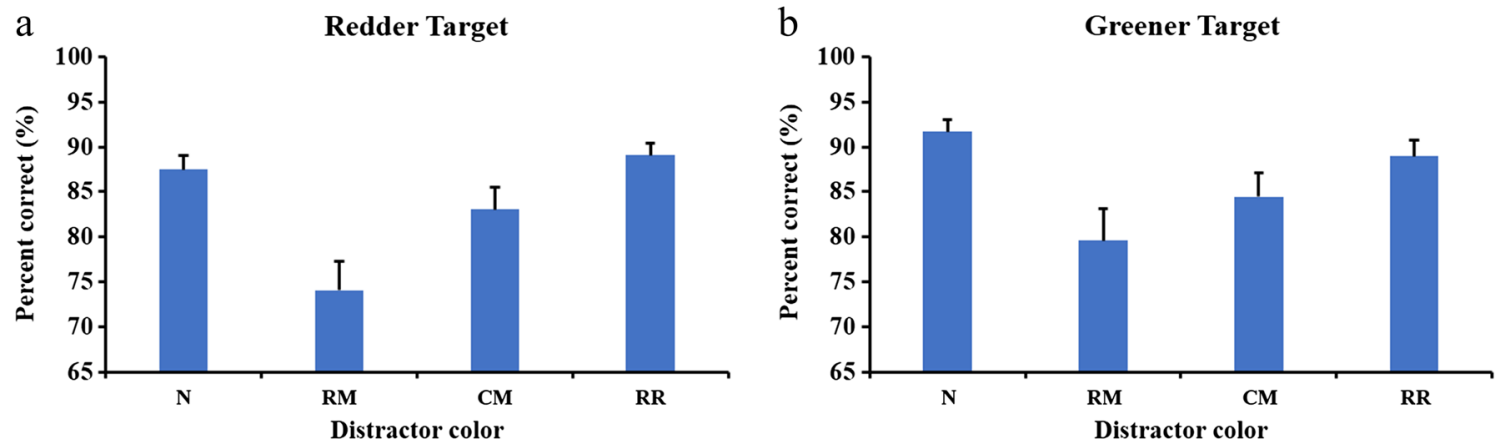

Fig. 4 Accuracy of different distractor conditions for the redder target (a) and greener target (b) in Experiment 2. Error bars represent the standard error 
Since the best relation-matched distractors robustly captured attention more than the color-matched distractors when distractors appeared outside of the attentional window, relation-based attention shares the same characteristic with the feature-based attention in the global selection mechanism. Given that both relation-based and featurebased capture showed global selection, the dissociation between them in the previous studies ( $\mathrm{Du} \&$ Jiao, 2016; Du et al., 2014) might be due to a strategical choice of two attentional mechanisms instead of the functional difference between them. Previous studies showed that features-based selection occurred outside of the attentional window, but the relation-based selection occurred within the attentional window when participants needed to find a particular target and knew both the target-defining feature and the target-nontarget relation (Du \& Jiao, 2016; Du et al., 2014). However, when there were multiple target colors and participants were instructed to search targets based on the target-nontarget relation, relationmatched distractor captured attention more than the features-matched distractor outside of the attentional window in both experiments of the present study. Especially since both relation-based and feature-based strategies can be used in Experiment 2, relation-matched distractors still robustly captured the most attention. Thus, we argued that both random selection of multiple target colors and explicit instruction might account for the preference of relation-based selection outside of the attentional window.

Both experiments also showed that the redder target block had lower accuracy than the greener target block. One possible explanation is that people are more sensitive to green than to red color (Kelly, 1973, 1974). Thus, greener targets are more likely to be identified than redder targets. Alternatively, it might be due to the more pronounced contrast between greenish targets and their distractors than to reddish targets and their distractors. These possibilities need further examination.

In sum, both experiments suggested that relation-based attention operates globally, as feature-based attention does. The dissociation between relation-based and feature-based attention might be a strategic choice of visual attention under different circumstances.

Acknowledgements This study was supported by grants from the National Natural Science Foundation of China (31470982) and the scientific foundation of the Institute of Psychology, Chinese Academy of Sciences (Y4CX033008).

Author contributions The study was conceived by F. Du. H. Hua, J. Zhang, and Y. Li collected and analyzed data under F. Du's supervision. F. Du, and H. Hua, and J. Zhang wrote the manuscript. H. Hua and J. Zhang contributed equally to this study.

Publisher's note Springer Nature remains neutral with regard to jurisdictional claims in published maps and institutional affiliations.

\section{References}

Becker, S. I. (2010). The role of target-distractor relationships in guiding attention and the eyes in visual search. Journal of Experimental Psychology: General, 139(2), 247-265.

Becker, S. I., Folk, C. L., \& Remington, R. W. (2010). The role of relational information in contingent capture. Journal of Experimental Psychology: Human Perception and Performance, 36 (6), 14601476.

Becker, S. I., Folk, C. L., \& Remington, R. W. (2013). Attentional capture does not depend on feature similarity, but on target-nontarget relations. Psychological Science, 24(5), 634-647.

Becker, S. I., Harris, A. M., Venini, D., \& Retell, J. D. (2014). Visual search for color and shape: When is the gaze guided by feature relationships, when by feature values? Journal of Experimental Psychology: Human Perception and Performance, 40(1), 264-291.

Brand, J., Oriet, C., Johnson, A. P., \& Wolfe, J. M. (2014). Flexible cue combination in the guidance of attention in visual search. Acta Psychologica, 153, 129-138.

Du, F., \& Abrams, R. A. (2008). Synergy of stimulus-driven salience and goal-directed prioritization: Evidence from the spatial blink. Perception \& Psychophysics, 70(8), 1489-1503.

Du, F., \& Abrams, R. A. (2010). Visual field asymmetry in attentional capture. Brain and Cognition, 72(2), 310-316.

Du, F., \& Abrams, R. A. (2012). Out of control: Attentional selection for orientation is thwarted by properties of the underlying neural mechanisms. Cognition, 124(3), 361-366.

$\mathrm{Du}, \mathrm{F} .$, \& Jiao, J. (2016). Feature-based attention is functionally distinct from relation-based attention: The double dissociation between color-based capture and color-relation-based capture of attention. Journal of Experimental Psychology: Human Perception and Performance, 42(4), 480-493.

Du, F., Yin, Y., Qi, Y., \& Zhang, K. (2014). Contingent orienting or contingent capture: A size singleton matching the target-distractor size relation cannot capture attention. Psychonomic Bulletin \& Review, 21(4), 1011-1018.

Eimer, M., \& Kiss, M. (2008). Involuntary attentional capture is determined by task set: Evidence from event-related brain potentials. Journal of Cognitive Neuroscience, 20(8), 1423-1433.

Folk, C. L., Leber, A. B., \& Egeth, H. E. (2002). Made you blink! Contingent attentional capture produces a spatial blink. Perception \& Psychophysics, 64(5), 741-753.

Folk, C. L., \& Remington, R. (1998). Selectivity in distraction by irrelevant featural singletons: Evidence for two forms of attentional capture. Journal of Experimental Psychology: Human Perception and Performance, 24(3), 847-858.

Folk, C. L., Remington, R. W., \& Johnston, J. C. (1992). Involuntary covert orienting is contingent on attentional control settings. Journal of Experimental Psychology: Human Perception and Performance, 18(4), 1030-1044.

Folk, C. L., Remington, R. W., \& Johnston, J. C. (1993). Contingent attentional capture: A reply to Yantis (1993). Journal of Experimental Psychology: Human Perception and Performance, 19(3), 682-685.

Goldstein, R. R., \& Beck, M. R. (2016). Inattentional blindness: A combination of a relational set and a feature inhibition set? Attention Perception \& Psychophysics, 78(5), 1245-1254.

Harris, A. M., Remington, R. W., \& Becker, S. I. (2013). Feature specificity in attentional capture by size and color. Journal of Vision, 13(3), 1-15.

Kelly, D. H. (1973). Lateral inhibition in human colour mechanisms. Journal of Physiology, 228(1), 55-72.

Kelly, D. H. (1974). Spatio-temporal frequency characteristics of colorvision mechanisms. Journal of the Optical Society of America, 64(7), 983-990. 
Kiss, M., \& Eimer, M. (2011). Attentional capture by size singletons is determined by top-down search goals. Psychophysiology, 48(6), 784-787.

Kiss, M., Grubert, A., \& Eimer, M. (2013). Top-down task sets for combined features: Behavioral and electrophysiological evidence for two stages in attentional object selection. Attention, Perception, \& Psychophysics, 75(2), $216-228$.

Meeter, M., \& Olivers, C. N. (2014). Target features and target-distractor relation are both primed in visual search. Attention, Perception, \& Psychophysics, 76(3), 682-694.
Serences, J. T., Shomstein, S., Leber, A. B., Golay, X., Egeth, H. E., \& Yantis, S. (2005). Coordination of voluntary and stimulus-driven attentional control in human cortex. Psychological Science, 16(2), 114-122.

Theeuwes, J. (1991). Exogenous and endogenous control of attention: The effect of visual onsets and offsets. Perception \& Psychophysics, 49(1), 83-90.

Treue, S., \& Martínez Trujillo, J. C. (1999). Feature-based attention influences motion processing gain in macaque visual cortex. Nature, 399, 575-579. 